\title{
Molecular Remodeling of Cardiac Mitochondria in Mice with Sepsis-Induced Cardiomyopathy
}

\section{Briana K. Shimada}

Translational Research Program, Department of Anesthesiology \& Center for Shock, Trauma and Anesthesiology Research, Baltimore, MD

\section{Liron Boyman}

University of Maryland School of Medicine, Baltimore, MD

\section{Jing Zhu}

Translational Research Program, Department of Anesthesiology \& Center for Shock, Trauma and Anesthesiology Research, Baltimore, MD

\section{Yang Yang}

Translational Research Program, Department of Anesthesiology \& Center for Shock, Trauma and Anesthesiology Research, Baltimore, MD

\section{Weiliang Huang}

University of Maryland School of Pharmacy, Baltimore, MD

\section{Maureen A. Kane}

University of Maryland School of Pharmacy, Baltimore, MD

\section{Nagendra Yadava}

Translational Research Program, Department of Anesthesiology \& Center for Shock, Trauma and Anesthesiology Research, Baltimore, MD

\section{Brian M. Polster}

Translational Research Program, Department of Anesthesiology \& Center for Shock, Trauma and Anesthesiology Research, Baltimore, MD

\section{Lin Zou}

Translational Research Program, Department of Anesthesiology \& Center for Shock, Trauma and Anesthesiology Research, Baltimore, MD

\section{W. Jonathan Lederer}

University of Maryland School of Medicine, Baltimore, MD

\section{Wei Chao ( $D$ wchao@som.umaryland.edu )}

Translational Research Program, Department of Anesthesiology \& Center for Shock, Trauma and Anesthesiology Research, Baltimore, MD

\section{Research Article}

Keywords: SIC, cardiomyopathy, ATP, muscle 
Posted Date: February 1st, 2021

DOl: https://doi.org/10.21203/rs.3.rs-149184/v1

License: (c) (i) This work is licensed under a Creative Commons Attribution 4.0 International License. Read Full License 


\section{Abstract}

Sepsis-induced cardiomyopathy (SIC) is a major contributing factor for morbidity and mortality in sepsis, but the underlying mechanisms are poorly understood. Previous work has suggested that mitochondrial oxidative phosphorylation is altered in mice with SIC, but the molecular mechanisms remain unidentified. Here, we performed a proteomic analysis of cardiac mitochondria isolated from sham and SIC mice. Of the 665 mitochondrial and metabolically coupled proteins identified in the proteomics assay, 36 were significantly altered in septic mice. The mitochondrial remodeling involved various energy metabolism pathways including subunits of the electron transport chain (ETC), fatty acid catabolism enzyme, and carbohydrate oxidative metabolism. We also identified a significant increase of pyruvate dehydrogenase (PDH) kinase 4 (PDK4) and inhibition of PDH activity in septic hearts. Furthermore, oxygen consumption by mitochondria from septic hearts was reduced when pyruvate was provided as a substrate but was unchanged in the presence of the Complex II substrate succinate, or the beta-oxidation substrate palmitoylcarnitine, both of which do not require PDH. Our data suggest that PDK4-mediated PDH inactivation and ETC molecular remodeling may contribute to the reduced mitochondrial oxidative metabolism during SIC and provide a molecular framework for future work.

\section{Introduction}

Sepsis-induced cardiomyopathy (SIC) is a common complication of sepsis that results in circulatory failure and contributes to high mortality ${ }^{1-4}$. While the etiology of SIC is complex and multifactorial, several studies provided evidence that dysfunction of the mitochondria plays a key role ${ }^{5,6}$. Yet, the molecular mechanisms underlying the mitochondrial dysfunction in the septic heart remain uncharacterized.

The mitochondria are the primary source of ATP to the working heart and encompass roughly 30 percent of the total volume of a heart muscle cell, the highest volume fraction of all mammalian cell types ${ }^{7}$. Daily energy consumption by the heart is the highest of all the organs ${ }^{8}$, requiring a constant and tightly regulated mitochondrial ATP production to meet these high energy demands ${ }^{9,10}$. Mitochondrial oxidative phosphorylation in the heart is fueled by fatty acid oxidation with additional contribution from the end product of carbohydrate catabolism, pyruvate. Fatty acids are metabolized by beta-oxidation while pyruvate is metabolized by pyruvate dehydrogenase (PDH). The metabolism of fatty acids and pyruvate inside the mitochondrial matrix yields reduced nicotinamide adenine dinucleotide (NADH) and reduced flavin adenine dinucleotide $\left(\mathrm{FADH} \mathrm{H}_{2}\right)$. NADH and $\mathrm{FADH}_{2}$ are the source of energy powering protons pumping out of the mitochondrial matrix by the electron transport chain (ETC) which generate a large electrochemical proton gradient across the mitochondrial inner membrane. This gradient energizes ATP production by ATP synthase (complex $\mathrm{V})^{11}$. The synthesized ATP then exits the mitochondrial matrix via the adenine nucleotide translocase (ANT1) and is used to fuel the production of contractile force, calcium signaling, and maintaining ionic gradients across sarcolemmal and organellar membranes. 
Several studies show that mitochondrial metabolic processes are altered in the hearts of mice with sepsis. Such findings include decreased oxygen consumption rate during active ATP production ('State III respiration'), reduced mitochondrial ATP synthesis, and reduced activities of several ETC complexes ${ }^{12-}$ 15. Furthermore, 24 hours after cecal ligation and puncture (CLP) in mice, ANT1 was found to be reduced in the left ventricle ${ }^{16}$. These multiple reports highlight the possible role played by mitochondrial dysfunction in SIC and thus the important need to understand better the underlying molecular mechanisms.

Here we employed a proteomics-based approach to examine the mitochondrial protein expression in the heart. Among 665 detected proteins with a known mitochondrial localization or mitochondrial association, 36 varied significantly between healthy and septic hearts. These included those with key functions in glucose and fatty acid metabolism, as well as subunits of the electron transport chain. Of particular interest, we found an increase in PDH kinase isozyme 4 (PDK4) in septic hearts, one of four isoforms with pyruvate dehydrogenase (PDH) as its primary target ${ }^{17,18}$. Phosphorylation of PDH by PDK4 leads to PDH inhibition ${ }^{17,19}$, which in turn, suppresses the entry of pyruvate into the Krebs cycle. The findings of this work provide a molecular framework toward a better understanding of mitochondrial dysfunction in SIC.

\section{Methods}

\section{Animals}

C57BL/ 6 mice were purchased from Jackson Laboratory (Bar Harbor, ME). Adult male mice were between 9-12 weeks of age and weighed between $20-30 \mathrm{~g}$. The animal protocols were approved by the Institutional Animal Care and Use Committee, University of Maryland School of Medicine (Baltimore, MD). All animal experiments were performed in compliance with the relevant guidelines and regulations of the National Institutes of Health (Bethesda, MD) and the ARRIVE guidelines of the National Centre for the Replacement, Refinement, and Reduction of Animals in Research (London, EN).

\section{Cecal ligation and puncture}

A clinically relevant mouse model of sepsis was generated by cecal ligation and puncture (CLP) surgery as described previously with minor modifications ${ }^{20-23}$. Briefly, mice were anesthetized with ketamine $(100 \mathrm{mg} / \mathrm{kg})$ and xylazine $(10 \mathrm{mg} / \mathrm{kg})$. The abdominal cavity was opened in layers and the feces were gently migrated to fill the distal part of the cecum. The cecum was ligated approximately $1.0 \mathrm{~cm}$ from the tip and was punctured with an 18-gauge needle. A small droplet of feces was extruded to ensure patency of the puncture site and the cecum was returned to the abdominal cavity. Sham-operated mice underwent laparotomy without CLP. The abdominal wall was closed in layers with $7-0$ sterile sutures. After surgery, pre-warmed saline (50 ml/kg body weight) was administered subcutaneously. Bupivacaine $(3 \mathrm{mg} / \mathrm{kg}$ ) and buprenorphine $(0.1 \mathrm{mg} / \mathrm{kg})$ were given post-operatively to manage pain.

\section{Echocardiography}


Echocardiography was performed in non-anesthetized mice as previously described ${ }^{20}$.

\section{Langendorff measurements}

Mice were heparinized (1000 IU/kg, intraperitoneal) and anesthetized with ketamine $(100 \mathrm{mg} / \mathrm{kg})$ and xylazine $(10 \mathrm{mg} / \mathrm{kg})$. Following confirmation of anesthesia, hearts were then excised and subjected to an ex vivo Langendorff perfusion model as previously described with minor modifications ${ }^{20}$. A water-filled balloon was introduced into the left ventricle to record LV pressure (PowerLab, AD Instruments, Denver, CO). Hearts were paced at $420 \mathrm{bpm}$ and left ventricular developed pressure (LVDP, left ventricular end systolic pressure - left ventricular end diastolic pressure, LVESP - LVEDP) was recorded continuously for 30 minutes. LVDP was taken as an average of the pressure over those 30 minutes.

\section{Isolation of cardiac mitochondria}

Mouse cardiac mitochondria were isolated as previously described ${ }^{24}$. A BCA assay was used to quantify the concentration of isolated mitochondria (in $\mathrm{mg}$ mitochondrial protein per $\mathrm{ml}$ ). The mitochondria were kept on ice and used for respiration measurements within 4 hours of isolation. The remaining mitochondria were stored at $-80^{\circ} \mathrm{C}$ for Western blots. Mitochondrial assay solution (MAS, $1 \mathrm{X}$ ) was comprised of: $70 \mathrm{mM}$ sucrose, $220 \mathrm{mM}$ mannitol, $10 \mathrm{mM} \mathrm{KH}_{2} \mathrm{PO}_{4}, 5 \mathrm{mM} \mathrm{MgCL}_{2}, 2 \mathrm{mM}$ HEPES, $1 \mathrm{mM}$ EGTA, $0.2 \%(w / v)$ fatty acid-free BSA $(\mathrm{pH}=7.2)^{25}$. Drugs and substrates were prepared as described previously and diluted to the following final concentrations: $4 \mathrm{mM}$ ADP, $2.5 \mu \mathrm{g} / \mathrm{mL}$ oligomycin, $4 \mu \mathrm{M}$ FCCP, $4 \mu \mathrm{M}$ antimycin A, $10 \mathrm{mM}$ succinate, $2 \mathrm{mM}$ malate, $2 \mu \mathrm{M}$ rotenone, and $10 \mathrm{mM}$ pyruvate ${ }^{25}$.

\section{RNA extraction and qRT-PCR}

Myocardial tissue RNA was extracted using TRIzol (Sigma) and quantified using the Nanodrop as reported previously ${ }^{22}$. The sequences of the primers used in the study are listed in Table 2.

\section{Western blot}

Whole hearts were washed in PBS and snap-frozen in liquid nitrogen. Isolated mitochondria were stored at $-80^{\circ} \mathrm{C}$. Both tissue and isolated mitochondria were then resuspended in NP-40 lysis buffer containing complete protease inhibitor cocktails (Roche Diagnostics, Indianapolis, IN) and protein concentrations were quantified using a BCA protein assay. Equivalent protein concentrations were fractionated by SDSPAGE under reducing conditions and blotted with the following antibodies: p-PDH ${ }^{\text {serine293 }}$ (Abcam, Cambridge, MA, 1:1000) and PDH antibody cocktail (Abcam, 1:1000), OXPHOS antibody rodent cocktail (Abcam, $6.0 \mu \mathrm{g} / \mathrm{mL}$ ), DLAT (Proteintech, Rosemont, IL, 1:2000), GAPDH (CST, Danvers, MA, 1:1000), SERCA2 (Santa Cruz, Dallas, TX, 1:200), and cytochrome C (BD, Franklin Lakes, NJ, 1:250). Whole hearts were used to assess $\mathrm{p}-\mathrm{PDH}$ and $\mathrm{PDH}$ levels while isolated mitochondria were used to determine the expression levels of complexes I-V. Both whole hearts and isolated mitochondria were used to determine levels of GAPDH, SERCA, and cytochrome $C$.

\section{Proteomics}


Mitochondria isolated from the hearts of sham or CLP mice were solubilized in 5\% sodium deoxycholate and were washed, reduced, alkylated and trypsinolyzed in a filter as previously described ${ }^{26,27}$. Tryptic peptides were separated on a nanoACQUITY UPLC analytical column (BEH130 C18, $1.7 \mu \mathrm{m}, 75 \mu \mathrm{m} \times 200$ $\mathrm{mm}$, Waters Corporation) over a 165-minute linear acetonitrile gradient (3-40\%) with $0.1 \%$ formic acid on a Waters nano-ACQUITY UPLC system and analyzed on a coupled Thermo Scientific Orbitrap Fusion Lumos Tribrid mass spectrometer as described ${ }^{28}$. Full scans were acquired at a resolution of 240,000, and precursors were selected for fragmentation by collision-induced dissociation (normalized collision energy at $35 \%$ ) for a maximum 3-second cycle. Tandem mass spectra were searched against a UniProt mouse reference proteome using Sequest HT algorithm ${ }^{29}$ and MS Amanda algorithm ${ }^{30}$ with a maximum precursor mass error tolerance of $10 \mathrm{ppm}$. Carbamidomethylation of cysteine and deamidation of asparagine and glutamine were treated as static and dynamic modifications, respectively. Resulting hits were validated at a maximum false discovery rate of 0.01 using a semi-supervised machine learning algorithm Percolator ${ }^{31}$. Label-free quantifications were performed using Minora, an aligned AMRT (Accurate Mass and Retention Time) cluster quantification algorithm (Thermo Scientific, 2017). Protein abundance ratios between the CLP and the sham were measured by comparing the MS1 peak volumes of peptide ions, whose identities were confirmed by MS2 sequencing as described above.

\section{Seahorse Extracellular Flux (XF) assay}

All XF assays were performed using an XF24 as described previously with minor modifications ${ }^{25} .2 .5,5$, or $7.5 \mu \mathrm{g}$ of isolated cardiac mitochondria in a volume of $50 \mu \mathrm{L}$ were delivered to each well in quadruplicate. Mitochondria were then centrifuged at $2000 \mathrm{xg}$ for 20 minutes at $4^{\circ} \mathrm{C}$ in a swinging bucket microplate adapter to attach the mitochondria to the plate. After centrifugation, $625 \mu \mathrm{L}$ of warm $1 \mathrm{X}$ MAS + substrate was added to each well. Either the Complex I-linked substrates pyruvate $(10 \mathrm{mM})$ and malate ( $2 \mathrm{mM})$, the Complex II substrate succinate $(10 \mathrm{mM})$ in the presence of the Complex I inhibitor rotenone $(2.5 \mu \mathrm{M})$, or the beta-oxidation substrate palmitoylcarntine $(40 \mu \mathrm{M})$ and malate $(1 \mathrm{mM})$ were given to the mitochondria in the $1 \mathrm{X}$ MAS. The plate was then allowed to equilibrate at $37^{\circ} \mathrm{C}$ for 10 minutes and subsequently transferred to the XF24 instrument for oxygen consumption rate (OCR) measurements. All OCR data was normalized to $1 \mu \mathrm{g}$ of protein.

\section{PDH activity assay}

PDH activity was assessed using a commercially available PDH activity kit from Abcam (Catalog No: ab109902). Hearts were homogenized in ice-cold PBS containing protease and phosphatase inhibitors. Sample concentration was determined using a BCA protein assay and each sample was adjusted to 200 $\mu \mathrm{g}$ of protein. Assay was then performed to the manufacturer's specifications. Plate was read at $450 \mathrm{~nm}$ in kinetic mode for 15 minutes at room temperature. The rate $(\Delta \mathrm{mOD} / \mathrm{min})$ was then calculated by using

$$
\frac{\text { Rate }=\left(\text { Absorbance }_{t 2}-\text { Absorbance }_{t 1}\right)}{\text { time }(\text { minutes })} \text {. }
$$

\section{Statistical analysis}


Statistical analysis was performed using GraphPad Prism 8 software (GraphPad, La Jolla, Ca). Unless stated otherwise, the distributions of the continuous variables were expressed as the mean \pm SEM.

Proteomic comparisons between the CLP mice and the sham were performed by FDR corrected, one-way ANOVA test and z-score. The statistical significance of the difference between groups was measured by $\mathrm{t}$ test or one-way ANOVA with Tukey's post hoc test or two-way ANOVA with Sidak's post hoc test for more than two groups. The null hypothesis was rejected for $P<0.05$ with the two-tailed t test.

\section{Results}

\section{Polymicrobial sepsis induces significant cardiac dysfunction}

To model sepsis-induced cardiomyopathy (SIC), we subjected mice to cecal ligation and puncture (CLP) or sham laparotomy. Cardiac function was assessed at baseline prior to surgery and again at 6- and 24hours post-surgery using echocardiography (Fig. 1A-B). We observed significantly decreased cardiac output, stroke volume, heart rate, LVIDd, and percent fractional shortening in the mice that underwent CLP surgery compared with sham (Fig. 1C). To rule out possible in vivo confounders associated with the loaddependent echocardiographic measurements, we isolated hearts from sham and CLP mice 24 hours after procedures and subjected the mouse hearts to ex vivo Langendorff apparatus. Analysis of these hearts revealed a significant decrease in left ventricular developed pressure (LVDP) in the CLP group compared with sham ( $104.4 \pm 12.9$ vs. $54.3 \pm 9.9 \mathrm{mmHg}$, sham vs. CLP, $n=3-4, P<0.01)$ as well as in $\mathrm{dP} / \mathrm{dt}$ maximum (3852 \pm 416 vs. $2010 \pm 341 \mathrm{mmHg} / \mathrm{s})$ and minimum $(-2591 \pm 293$ vs. $-1376 \pm 241 \mathrm{mmHg} / \mathrm{s}$, sham vs. CLP $n=$ $3-4, P<0.01$, Fig. 1D). These data established that CLP mice developed intrinsic cardiomyopathy that was independent of preload conditions.

\section{Proteomic analysis reveals altered mitochondrial metabolic proteins in septic heart}

One of the molecular mechanisms behind the contractile dysfunction during sepsis is the impairment of mitochondrial bioenergetics. To determine whether altered mitochondrial protein expression is associated with bioenergetic impairment and subsequent myocardial dysfunction, we isolated cardiac mitochondria from sham mice and CLP mice exhibiting clear cardiac dysfunction as measured by echocardiography ( $n=6 /$ group). The mitochondria were then evaluated using liquid chromatography-tandem mass spectrometry. Of the 665 mitochondrial and metabolically coupled proteins with an established physical or functional mitochondrial association that were identified in the proteomics assay, 36 were found to be significantly altered between sham and CLP mitochondria (Fig. 2A). A complete list of the 36 proteins can be found in Table 1. Notably, these 36 mitochondrial and metabolically coupled proteins differentially expressed in the septic hearts are reportedly involved in various bioenergetic functions such as pyruvate metabolism, fatty acid metabolism, electron transport, and mitochondrial membrane integrity (Fig. 2B, Table 2). Pyruvate dehydrogenase kinase 4 (PDK4), a primary regulator of PDH, was significantly upregulated in the mitochondrial fraction isolated from septic hearts. Pyruvate kinase (PK), the enzyme responsible for catalyzing the transfer of a phosphate group from phosphoenolpyruvate (PEP) to adenosine diphosphate (ADP) to yield pyruvate and one molecule of ATP in the last step of glycolysis was also upregulated. Meanwhile, methylmalonyl-CoA epimerase (MCE), an enzyme involved in odd- 
chain fatty acid catabolism, was significantly down-regulated in sepsis heart mitochondria (Fig. 2C). In addition, we discovered two other proteins indirectly related to fatty acid metabolism, phytanol-CoA dioxygenase (PHYH), and carbonyl reductase [NADPH] 2 (CBR2), to be up-regulated. PHYH catalyzes the first step in the alpha-oxidation of phytanic acid while CBR2 metabolizes aldehydes and ketones derived from lipid peroxidation (Fig. 2C). Moreover, electron transport chain proteins, including NADH dehydrogenase [ubiquinone] 1 beta subcomplex subunit 8 (NDUFB8, a subunit of Complex I), cytochrome c oxidase 5B (COX5B, a subunit of Complex IV), cytochrome c oxidase copper chaperone (COX17, a nuclear-encoded protein essential for the assembly of Complex IV), were downregulated, while NADPHcytochrome P450 reductase (P450R, an enzyme required for the transfer of electrons from NADP to cytochrome P450), was up-regulated in the mitochondria of septic mice (Fig. 2D).

\section{Cardiac PDH activity is inhibited and corresponds with an increase in PDK4 and phosphorylation of PDH in septic heart}

We performed qRT-PCR to test whether the observed increase in mitochondrial PKD4 protein expression in septic hearts was due to increased transcription. We found a 6.8-fold increase in PDK4 gene expression in the cardiac tissue of septic mice as compared to sham mice (Fig. 3A, $P<0.001$ ). In contrast, there was no difference in the gene expression of PDH E1 a subunit between sham and sepsis hearts (Fig. 3B). Since the primary role of PDK4 is to phosphorylate the PDH E1 subunit, which is known to inhibit PDH enzyme activity, we performed Western blots using whole heart tissue from sham and CLP mice and found a significant increase in phosphorylated PDH at serine-293 in septic hearts (Fig. 3C-D). We then assessed

whether these molecular modifications resulted in a functional decrease of PDH activity using a commercially available PDH activity assay kit. We discovered septic hearts had markedly lower PDH activity than sham hearts, $(27.4 \pm 2.4$ vs. $6.5 \pm 4.2 \Delta \mathrm{mOD} / \mathrm{min}$, sham vs. CLP, $n=5, P<0.0001)$ (Fig. $3 \mathrm{E}-\mathrm{F})$.

\section{Complexes I-V are downregulated in septic mitochondria}

To confirm the downregulation of the ETC Complex I subunit NDUFB8 and the Complex IV subunit COX5B and COX17 as revealed by proteomics in septic mitochondria, we used an antibody cocktail to detect representative subunits of all five oxidative phosphorylation complexes in isolated mitochondria. Densitometric quantification of Western blots revealed that all five representative proteins were significantly decreased in septic mitochondria (Fig. 4A-F) with a 1.25-, 3.11-, 1.44-, 1.14-, and 1.17-fold decrease in Complex I, II, III, IV, and V subunits, respectively. In contrast and consistent with the proteomics data, there was no difference in the expression of DLAT (PDH E2 subunit) between the sham and sepsis mitochondria (Fig. 4G). To confirm the purity of the isolated mitochondria, we blotted mitochondrial proteins for GAPDH and SERCA, two proteins highly expressed in the cytosol and the sarcoplasmic reticulum, respectively, to ensure mitochondrial separation from other cellular compartments. We found very minimal GAPDH presence and no SERCA2 presence in the isolated mitochondria (data not shown).

Cardiac mitochondria of sepsis mice show a decreased ADP-stimulated oxygen consumption rate when given pyruvate and malate as substrates prior to extracellular flux assay 
Next, we assessed mitochondrial oxygen consumption rate (OCR) using a Seahorse Extracellular Flux assay. Isolated mitochondria were fueled by the Complex I-linked substrates pyruvate and malate, the Complex II substrate succinate in the presence of the Complex I inhibitor rotenone (Fig. 5A-B), or by the beta-oxidation substrate palmitoylcarnitine. We found that relative to sham, mitochondria isolated from septic hearts showed lower basal OCR $\left(15.89 \pm 4.89\right.$ vs. $9.37 \pm 5.32 \mathrm{pmol} \mathrm{O}_{2} / \mathrm{min} / \mu \mathrm{g}$, sham vs. CLP, $n=9, P$ $<0.05$ ) (Fig. 5C), and state 3 respiration (108.56 \pm 19.71 vs. $86.45 \pm 14.91 \mathrm{pmol} \mathrm{O}_{2} / \mathrm{min} / \mu \mathrm{g}$, sham v. CLP, $n$ $=9, P<0.05)$ (Fig. 5D), when given pyruvate and malate as substrates. States IV and III $\mathrm{u}_{\mathrm{u}}$ were unchanged (Fig. 5E-F). However, no difference in OCR was observed when mitochondria were energized by succinate or when mitochondrial ATP production was fueled by palmitoylcarnitine (Fig. 5G-N). The decreased OCR in the presence of pyruvate and malate but not Complex II or beta-oxidation substrates suggests that CLP mitochondria have reduced capacity to metabolize pyruvate. Therefore, these finding suggest that in sepsis, there is a reduced capacity to utilize pyruvate, likely due to inactivation of PDH by PDK4.

\section{Discussion}

In a mouse model of polymicrobial sepsis, we demonstrated sepsis-induced cardiomyopathy with significant contractile dysfunction as illustrated by the echocardiographic evidence in vivo and in isolated Langendorff-perfused hearts. To determine the impact of sepsis on mitochondrial proteins in the heart, we carried out a quantitative proteomic analysis on mitochondria isolated from septic and sham mice, which revealed a major molecular remodeling in the mitochondria of septic heart. At 24 hours after CLP, PDK4, the primary kinase that inactivates PDH was upregulated whereas other critical metabolic enzymes were reduced, including the ETC proteins, NDUFB8, COX5B, and COX17. Notably, the upregulation of PDK4 was associated with a significant rise of phosphorylation of the PDH E1 subunit at serine-293. These molecular changes were associated with marked inhibition of PDH activity in septic hearts. Functionally, OCR by mitochondria from septic hearts were decreased as compared with the sham controls when respiration was fueled by pyruvate. However, OCR did not differ when succinate was used to measure respiration that is dependent on Complex II and downstream components of the ETC or when the beta-oxidation substrate palmitoylcarnitine was used.

Molecular changes to PDH and complex I during sepsis

PDH activity is an overall rate-limiting step in oxidative phosphorylation that is powered by pyruvate. Therefore, the reduced OCR when oxidative phosphorylation was fueled by pyruvate and malate is consistent with a potential mechanism of PDK4-dependent PDH inhibition that is supported by our proteomics findings and PDH activity assay in septic hearts. Notably, we did not find compensatory upregulation of beta-oxidation suggesting that PDH inactivation is through PDK4 and not reciprocal regulation by fatty acid oxidation. Other studies did find compensatory upregulation of beta-oxidation in genetic mouse models where PDH was downregulated ${ }^{32,33}$. These findings appear to support the idea that molecular downregulation of PDH does not involve a switch in metabolic fuel selection but rather decreases the overall capacity of the mitochondria to utilize metabolic substrates to power the production of ATP. Our findings also suggest that despite the observed downregulation of subunits from all four ETC 
complexes, consistent with prior evidence for diminished ETC activity in sepsis models ${ }^{15,34-37}$, decreased electron flux through Complex I is likely responsible for the reduced functional capacity of the cardiac mitochondria from septic mice. The decreased flux could be due to impaired NADH regeneration, decreased Complex I expression, or a combination of the two. Supporting this notion is the proteomics data that demonstrated a significant decrease in NADH dehydrogenase 1 beta subcomplex subunit 8 (NDUFB8), a Complex I accessory subunit. Several studies suggested that Complex I activity is reduced during sepsis in the heart and the brain, including one study that utilized isolated cardiac mitochondria from a CLP sepsis model ${ }^{15,34,35,37}$. However, these studies only reported that the enzymatic activity of Complex I was decreased and did not assess the functional ability of the mitochondria to utilize Complex I-linked substrates that depend on the activity of Krebs cycle dehydrogenases, including PDH. It is probable that both PDH inactivation and Complex I downregulation contribute to the reduced ability of the mitochondria in sepsis to utilize pyruvate and malate for respiration. All together, these data provide additional molecular insights into the previously suggested role of glycolysis in sepsis-induced cardiomyopathy and lactic acidosis ${ }^{38}$.

Use of CLP mode and isolated mitochondria in the study of septic cardiomyopathy

CLP is one of the most common models of polymicrobial sepsis. It simulates acute peritonitis following bowel ischemia and perforation in humans. While it produces many clinical presentations similar to human sepsis, such as cytokine storm, circulatory shock, cardiac depression, and acute kidney injury, the model is highly heterogeneous in its severity and organ dysfunction. In this study, some, but not all CLP mice, developed cardiac dysfunction. To reduce the heterogeneity and to link the physiological phenotype to the bioenergetics analysis, we only selected those septic mice that had demonstrated cardiac dysfunction by echocardiography for the subsequent mitochondrial proteomics analysis and mitochondrial functional tests. With this approach, we enriched for mitochondrial proteins, as we have reported recently ${ }^{24}$, for mass spec analysis and mitochondrial specific molecules and functions. It should be noted that in our protocol, we take additional steps that are not routinely used to increase purity but with the cost of lower yield. We pellet the non-mitochondrial fractions at low-speed centrifugation of $600 \mathrm{xg}$ three times where typically this is only done once. To pellet and isolate mitochondria, we centrifuge our samples at $3200 \mathrm{xg}$ instead of the more commonly used $10,000 \mathrm{xg}$. In addition, after we obtain the mitochondrial pellet, we repeat this step three more times to further discard any possible cytosolic or other soluble materials of the supernatant, whereas typical isolation protocols utilize only one cycle. As result, the mitochondrial preparations contain only trace amounts of GAPDH and SERCA, two proteins highly expressed in the cytoplasma and the sarcoplasmic reticulum, respectively. All together, these data suggest that the cardiac mitochondrial preparations in our study are highly pure and with minimal cytosolic or other organelle contamination.

Proteomic analysis of mitochondria from septic hearts

The proteomic analysis identified a total of 1135 proteins, 665 of which appeared to be mitochondria or metabolically coupled proteins. It should be noted that while proteins belonging to all four ETC 
complexes were detected in both sham and CLP cardiac mitochondria, only Complex I and Complex IV subunits exhibited differential expression between sham and CLP mice. Further analysis by Western blot, however, revealed that representative subunits from all four ETC complexes and well as the ATP synthase were downregulated to different degrees. In addition, we found other proteins involved in pyruvate metabolism, fatty acid metabolism, and electron transport to be perturbed in mitochondria isolated from the septic hearts. Notably, enzymes traditionally involved in beta-oxidation did not show altered expression, consistent with our functional OCR data using palmitoylcarnitine. Therefore, the functional significance of the proteomic findings of these enzymes, i.e., methylmalonyl-CoA epimerase (MCE), phytanol-CoA dioxygenase (PHYH), and carbonyl reductase [NADPH] 2 (CBR2) remains to be investigated. MCE catabolizes fatty acids with odd-length carbon chains. $\mathrm{PHYH}$ is a peroxisomeassociated protein involved in alpha-oxidation of fatty acids, and CBR2 metabolizes aldehydes and ketones derived from lipid peroxidation ${ }^{39}$. Lipid peroxidation occurs when free radicals gain electrons from lipids, causing the formation of fatty acid radicals. As sepsis is known to generate high levels of reactive oxygen species (ROS) 40,41 , it is speculated that these mitochondria have fatty acid radicals, resulting in an increased need for CBR2-dependent detoxification. Moreover, we did not see a sepsisinduced reduction in the adenine nucleotide translocator (ANT1) in cardiac mitochondria, in contrast to a report by Pan S., et. al ${ }^{16}$. However, we isolated mitochondria from the whole heart, whereas that study focused on the left ventricle. Furthermore, as noted earlier, the CLP model itself is highly heterogeneous and may also account for why comparative proteomics did not find decreased ANT levels. Overall, the quantitative proteomics analysis revealed several metabolic pathways that were disturbed in CLP mitochondria, providing the molecular basis for additional explorations of the mechanisms of mitochondrial dysfunction in sepsis-induced cardiomyopathy.

Pyruvate dehydrogenase inactivation during sepsis

One of the main findings of this study was of the phosphorylation and subsequent inactivation of PDH in the heart during sepsis. This change is consistent with data from other cells/tissues, e.g., in skeletal muscle and peripheral blood mononuclear cells, that PDH has decreased activity during sepsis ${ }^{42-46}$. Using a chronic sepsis model induced by intraabdominal placement of a fecal-agar pellet containing $E$. coli and $B$. fragilis in rats, Vary and co-workers demonstrated PDH inactivation in skeletal muscle after 5 days. The specific PDK isoform responsible for PDH inactivation was not identified in these prior studies. Our data suggest that in the heart, PDH inactivation happens earlier in the development of sepsis, and given the detection of increased PDK4 mRNA and protein, is likely a transcriptionally regulated event. Thus, the bioenergetic changes to mitochondria from sepsis hearts may be due, at least in part, to molecular remodeling rather than non-specific damage. A better understanding of these mitochondrial remodeling events may provide new targets for intervention, e.g., reducing lactic acidosis to mitigate cardiomyopathy.

Limitations of the current study 
There were several limitations in the study. For one, we did not verify the changes of all 36 of the differentially expressed proteins that were found in our proteomics data by Western blot, as we elected to focus more on PDH and overall mitochondria function. We also did not test all of the proteins found by the proteomics and therefore, there are probably several other metabolically-related proteins that may be involved in the cardiac energy deficit during sepsis. It must be stressed that molecular remodeling, as identified by mass spec, even when confirmed by other molecular tests such as Western blot, does not necessarily imply functional impairment. More comprehensive function testing of the metabolic pathways identified by the proteomics data will be pursued in our future studies.

Summary and conclusions

As illustrated in Fig. 6, employing a proteomics approach, we identified 36 proteins, either mitochondrial proteins or metabolically coupled, with differential expression in cardiac mitochondria from mice with SIC. Among them, notably, PDK4 was upregulated at both mRNA and protein levels, along with an increase in the phosphorylated PDH and marked reduction in PDH activity in septic heart. These molecular remodeling led to reduced mitochondrial capability to utilize pyruvate in the septic heart. These findings provide a molecular framework to better understanding the etiology of sepsis and sepsis-induced cardiomyopathy.

\section{Declarations}

\section{Acknowledgements}

This work was supported by NIH grants R01GM117233, R01GM122908, R01NS110567 (to W.C.), R35GM124775 and R01NS110567 (to L.Z.), and by Frontiers in Anesthesia Research Award from International Anesthesia Research Society (to W.C.), and also supported by the American Heart Association Scientist Development Grant Award 15SDG22100002 (to L.B.) and by NIH grants R01HL142290, U01HL116321, R01HL140934, R01AR071618 (to W.J.L.). Additional support was provided by the University of Maryland School of Pharmacy Mass Spectrometry Center (SOP1841-IQB2014) (to W.H. and M.A.K.).

\section{Author contributions}

B.K.S., L.B., W.J.L., W.C. designed the study, analyzed the data, and wrote the manuscript. B.K.S., L.B., J.Z., Y.Y., W.H. performed the experiments. M.A.K., N.Y., B.M.P., L.Z. participated in interpretation of the data and revision of the manuscript. B.K.S, L.B., W.C. finalized the manuscript. All authors reviewed the manuscript.

\section{Competing interests}

The authors declare no competing interests. 


\section{References}

1. Cimolai, M. C., Alvarez, S., Bode, C. \& Bugger, H. Mitochondrial Mechanisms in Septic Cardiomyopathy. Int J Mol Sci.16, 17763-17778 https://doi.org/10.3390/ijms160817763 (2015).

2. Court, O., Kumar, A., Parrillo, J. E. \& Kumar, A. Clinical review: Myocardial depression in sepsis and septic shock. Crit Care.6, 500-508 https://doi.org/10.1186/cc1822 (2002).

3. Stanzani, G., Duchen, M. R. \& Singer, M. The role of mitochondria in sepsis-induced cardiomyopathy. Biochim Biophys Acta Mol Basis Dis.1865, 759-773 https://doi.org/10.1016/j.bbadis.2018.10.011 (2019).

4. Wilhelm, J. et al. Severity of cardiac impairment in the early stage of community-acquired sepsis determines worse prognosis. Clin Res Cardiol.102, 735-744 https://doi.org/10.1007/s00392-0130584-z (2013).

5. Romero-Bermejo, F. J., Ruiz-Bailen, M., Gil-Cebrian, J. \& Huertos-Ranchal, M. J. Sepsis-induced cardiomyopathy. Curr Cardiol Rev.7, 163-183 https://doi.org/10.2174/157340311798220494 (2011).

6. Rudiger, A. \& Singer, M. Mechanisms of sepsis-induced cardiac dysfunction. Crit Care Med.35, 15991608 https://doi.org/10.1097/01.CCM.0000266683.64081.02 (2007).

7. Barth, E., Stammler, G., Speiser, B. \& Schaper, J. Ultrastructural quantitation of mitochondria and myofilaments in cardiac muscle from 10 different animal species including man. J Mol Cell Cardiol.24, 669-681 https://doi.org/10.1016/0022-2828(92)93381-s (1992).

8. Briese, V., Than, G., Richter, D., lino, K. \& Seppala, M. [Placental protein 5 and pregnancy zone protein in ovarian cysts and benign cystic ovarian tumors]. Zentralbl Gynakol.110, 821-823 (1988).

9. Brown, D. A. et al. Expert consensus document: Mitochondrial function as a therapeutic target in heart failure. Nat Rev Cardiol.14, 238-250 https://doi.org/10.1038/nrcardio.2016.203 (2017).

10. Wescott, A. P., Kao, J. P. Y., Lederer, W. J. \& Boyman, L. Voltage-energized Calcium-sensitive ATP Production by Mitochondria. Nat Metab.1, 975-984 https://doi.org/10.1038/s42255-019-0126-8 (2019).

11. Nicholls, D. G. \& Ferguson, S. J. Bioenergetics. Fourth edition / edn(Academic Press, Elsevier, 2013).

12. Chopra, M. et al. Modulation of myocardial mitochondrial mechanisms during severe polymicrobial sepsis in the rat. PLoS One.6, e21285 https://doi.org/10.1371/journal.pone.0021285 (2011).

13. Reynolds, C. M. et al. Nitric oxide synthase-2 induction optimizes cardiac mitochondrial biogenesis after endotoxemia. Free Radic Biol Med.46, 564-572

https://doi.org/10.1016/j.freeradbiomed.2008.11.007 (2009).

14. Kozlov, A. V. et al. Different effects of endotoxic shock on the respiratory function of liver and heart mitochondria in rats. Am J Physiol Gastrointest Liver Physiol.290, G543-549 https://doi.org/10.1152/ajpgi.00331.2005 (2006).

15. Vanasco, V. et al. Cardiac mitochondrial biogenesis in endotoxemia is not accompanied by mitochondrial function recovery. Free Radic Biol Med.77, 1-9 
https://doi.org/10.1016/j.freeradbiomed.2014.08.009 (2014).

16. Pan, S., Wang, N., Bisetto, S., Yi, B. \& Sheu, S. S. Downregulation of adenine nucleotide translocator 1 exacerbates tumor necrosis factor-alpha-mediated cardiac inflammatory responses. Am J Physiol Heart Circ Physiol.308, H39-48 https://doi.org/10.1152/ajpheart.00330.2014 (2015).

17. Bowker-Kinley, M. M., Davis, W. I., Wu, P., Harris, R. A. \& Popov, K. M. Evidence for existence of tissuespecific regulation of the mammalian pyruvate dehydrogenase complex. Biochem J.329 (Pt 1), 191196 https://doi.org/10.1042/bj3290191 (1998).

18. Korotchkina, L. G. \& Patel, M. S. Site specificity of four pyruvate dehydrogenase kinase isoenzymes toward the three phosphorylation sites of human pyruvate dehydrogenase. J Biol Chem.276, 3722337229 https://doi.org/10.1074/jbc.M103069200 (2001).

19. Dyck, J. R. et al. Absence of malonyl coenzyme A decarboxylase in mice increases cardiac glucose oxidation and protects the heart from ischemic injury. Circulation.114, 1721-1728 https://doi.org/10.1161/CIRCULATIONAHA.106.642009 (2006).

20. Zou, L. et al. Toll-like receptor 2 plays a critical role in cardiac dysfunction during polymicrobial sepsis. Crit Care Med.38, 1335-1342 https://doi.org/10.1097/CCM.0b013e3181d99e67 (2010).

21. Rittirsch, D., Huber-Lang, M. S., Flierl, M. A. \& Ward, P. A. Immunodesign of experimental sepsis by cecal ligation and puncture. Nat Protoc.4, 31-36 https://doi.org/10.1038/nprot.2008.214 (2009).

22. Zou, L., Feng, Y., Zhang, M., Li, Y. \& Chao, W. Nonhematopoietic toll-like receptor 2 contributes to neutrophil and cardiac function impairment during polymicrobial sepsis. Shock.36, 370-380 https://doi.org/10.1097/SHK.0b013e3182279868 (2011).

23. Zou, L. et al. Complement factor $B$ is the downstream effector of TLRs and plays an important role in a mouse model of severe sepsis. J Immunol.191, 5625-5635 https://doi.org/10.4049/jimmunol.1301903 (2013).

24. Wescott, A. P., Kao, J. P. Y., Lederer, W. J. \& Boyman, L. Voltage-energized calcium-sensitive ATP production by mitochondria. Nature Metabolism.1, 975-984 https://doi.org/10.1038/s42255-0190126-8 (2019).

25. Rogers, G. W. et al. High throughput microplate respiratory measurements using minimal quantities of isolated mitochondria. PLoS One.6, e21746 https://doi.org/10.1371/journal.pone.0021746 (2011).

26. Wisniewski, J. R., Zougman, A., Nagaraj, N. \& Mann, M. Universal sample preparation method for proteome analysis. Nat Methods.6, 359-362 https://doi.org/10.1038/nmeth.1322 (2009).

27. Erde, J., Loo, R. R., Loo, J. A. \& Enhanced FASP (eFASP) to increase proteome coverage and sample recovery for quantitative proteomic experiments. J Proteome Res.13, 1885-1895 https://doi.org/10.1021/pr4010019 (2014).

28. Williamson, J. C. et al. High-performance hybrid Orbitrap mass spectrometers for quantitative proteome analysis: Observations and implications. Proteomics.16, 907-914 https://doi.org/10.1002/pmic.201400545 (2016).

29. Eng, J. K., Fischer, B., Grossmann, J. \& Maccoss, M. J. A fast SEQUEST cross correlation algorithm. J Proteome Res.7, 4598-4602 https://doi.org/10.1021/pr800420s (2008). 
30. Dorfer, V. et al. MS Amanda, a universal identification algorithm optimized for high accuracy tandem mass spectra. J Proteome Res.13, 3679-3684 https://doi.org/10.1021/pr500202e (2014).

31. Kall, L., Canterbury, J. D., Weston, J., Noble, W. S. \& MacCoss, M. J. Semi-supervised learning for peptide identification from shotgun proteomics datasets. Nat Methods.4, 923-925 https://doi.org/10.1038/nmeth1113 (2007).

32. Kwong, J. Q. et al. The mitochondrial calcium uniporter underlies metabolic fuel preference in skeletal muscle. JCI Insight.3, https://doi.org/10.1172/jci.insight.121689 (2018).

33. Altamimi, T. R. et al. Cardiac-specific deficiency of the mitochondrial calcium uniporter augments fatty acid oxidation and functional reserve. J Mol Cell Cardiol.127, 223-231 https://doi.org/10.1016/j.yjmcc.2018.12.019 (2019).

34. Zang, Q. S. et al. Sepsis-induced cardiac mitochondrial dysfunction involves altered mitochondriallocalization of tyrosine kinase Src and tyrosine phosphatase SHP2. PLoS One.7, e43424 https://doi.org/10.1371/journal.pone.0043424 (2012).

35. Comim, C. M. et al. Mitochondrial respiratory chain and creatine kinase activities in rat brain after sepsis induced by cecal ligation and perforation. Mitochondrion.8, 313-318 https://doi.org/10.1016/j.mito.2008.07.002 (2008).

36. Yang, X., Lu, G. P., Cai, X. D., Lu, Z. J. \& Kissoon, N. Alterations of complex IV in the tissues of a septic mouse model. Mitochondrion.49, 89-96 https://doi.org/10.1016/j.mito.2018.11.008 (2019).

37. Jarkovska, D. et al. Cellular Mechanisms of Myocardial Depression in Porcine Septic Shock. Front Physiol.9, 726 https://doi.org/10.3389/fphys.2018.00726 (2018).

38. Zheng, Z. et al. Enhanced Glycolytic Metabolism Contributes to Cardiac Dysfunction in Polymicrobial Sepsis. J Infect Dis.215, 1396-1406 https://doi.org/10.1093/infdis/jix138 (2017).

39. Nakanishi, M., Deyashiki, Y., Ohshima, K. \& Hara, A. Cloning, expression and tissue distribution of mouse tetrameric carbonyl reductase. Identity with an adipocyte 27-kDa protein. Eur J Biochem.228, 381-387 (1995).

40. Zang, Q., Maass, D. L., Tsai, S. J. \& Horton, J. W. Cardiac mitochondrial damage and inflammation responses in sepsis. Surg Infect (Larchmt).8, 41-54 https://doi.org/10.1089/sur.2006.033 (2007).

41. Yao, X. et al. Mitochondrial ROS Induces Cardiac Inflammation via a Pathway through mtDNA Damage in a Pneumonia-Related Sepsis Model. PLoS One.10, e0139416 https://doi.org/10.1371/journal.pone.0139416 (2015).

42. Vary, T. C. \& Hazen, S. Sepsis alters pyruvate dehydrogenase kinase activity in skeletal muscle. $\mathrm{Mol}$ Cell Biochem.198, 113-118 https://doi.org/10.1023/a:1006993910781 (1999).

43. Nuzzo, E. et al. Pyruvate Dehydrogenase Activity Is Decreased in the Peripheral Blood Mononuclear Cells of Patients with Sepsis. A Prospective Observational Trial. Ann Am Thorac Soc.12, 1662-1666 https://doi.org/10.1513/AnnalsATS.201505-267BC (2015).

44. Vary, T. C. Sepsis-induced alterations in pyruvate dehydrogenase complex activity in rat skeletal muscle: effects on plasma lactate. Shock.6, 89-94 https://doi.org/10.1097/00024382-19960800000002 (1996). 
45. Vary, T. C. \& Martin, L. F. Potentiation of decreased pyruvate dehydrogenase activity by inflammatory stimuli in sepsis. Circ Shock.39, 299-305 (1993).

46. Alamdari, N. et al. Temporal changes in the involvement of pyruvate dehydrogenase complex in muscle lactate accumulation during lipopolysaccharide infusion in rats. J Physiol.586, 1767-1775 https://doi.org/10.1113/jphysiol.2007.149625 (2008).

\section{Tables}

Table 1. Proteomic analysis of cardiac mitochondrial proteins: 36 differentially expressed proteins between sham and CLP mice. 


\begin{tabular}{|c|c|c|c|c|}
\hline$\#$ & & Accession & $\begin{array}{l}\text { Gene } \\
\text { Name }\end{array}$ & $\begin{array}{l}\text { Abundance } \\
\text { Ratio } \\
\text { (CLP/sham) }\end{array}$ \\
\hline \multicolumn{5}{|c|}{ Pyruvate Metabolism } \\
\hline 1 & Pyruvate kinase & P52480 & PKM & 1.706 \\
\hline 2 & $\begin{array}{l}\text { Pyruvate dehydrogenase (acetyl-transferring)] } \\
\text { kinase isozyme } 4\end{array}$ & 070571 & PDK4 & 1.993 \\
\hline \multicolumn{5}{|c|}{ Fatty Acid Metabolism } \\
\hline 3 & Methylmalonyl-CoA epimerase & Q9D1I5 & MCEE & 0.595 \\
\hline 4 & Carbonyl reductase [NADPH] 2 & P08074 & CBR2 & 1.704 \\
\hline 5 & Phytanoyl-CoA dioxygenase, peroxisomal & 035386 & $\mathrm{PHYH}$ & 1.921 \\
\hline \multicolumn{5}{|c|}{ Lactate Production } \\
\hline 6 & L-lactate dehydrogenase $B$ chain & P16125 & LDHB & 1.481 \\
\hline 7 & L-lactate dehydrogenase & A0A1B0GSX0 & LDHA & 1.668 \\
\hline \multicolumn{5}{|c|}{ Electron Transport } \\
\hline 8 & $\begin{array}{l}\text { NADH dehydrogenase [ubiquinone] } 1 \text { beta } \\
\text { subcomplex subunit } 8 \text {, mitochondrial }\end{array}$ & Q9D6J5 & NDUFB8 & 0.592 \\
\hline 9 & Cytochrome $\mathrm{c}$ oxidase subunit $5 \mathrm{~B}$ & Q9D881 & GM11273 & 0.671 \\
\hline 10 & Cytochrome c oxidase copper chaperone & P56394 & $\operatorname{cox} 17$ & 0.609 \\
\hline 11 & NADPH--cytochrome $\mathrm{P} 450$ reductase & P37040 & P450R & 1.497 \\
\hline \multicolumn{5}{|c|}{ Mito Membrane Integrity } \\
\hline 12 & MICOS complex subunit MIC10 & Q7TNS2 & MINOS1 & 0.508 \\
\hline 13 & E3 ubiquitin-protein ligase MARCH5 & Q3KNM2 & MARCH5 & 2.685 \\
\hline \multicolumn{5}{|c|}{ Other } \\
\hline 14 & Glyceraldehyde-3-phosphate dehydrogenase & AOAOAOMQF6 & GAPDH & 1.660 \\
\hline 15 & Cathepsin D & P18242 & CTSD & 1.607 \\
\hline 16 & Mitochondrial brown fat uncoupling protein 1 & P12242 & UCP-1 & 2.410 \\
\hline 17 & Phosphate carrier protein & Q8VEM8 & SLC25A3 & 1.575 \\
\hline 18 & Cathepsin B & P10605 & CTSB & 1.777 \\
\hline 19 & Sideroflexin-3 & Q91V61 & SFXN3 & 7.061 \\
\hline 20 & Keratin, type II cytoskeletal 5 & Q922U2 & KRT5 & 2.413 \\
\hline
\end{tabular}




\begin{tabular}{|lllll|}
\hline 21 & B-cell receptor-associated protein 31 & Q61335 & BCAP31 & 1.631 \\
\hline 22 & 39S ribosomal protein L15 & Q9CPR5 & MRPL15 & 1.571 \\
\hline 23 & Transforming protein RhoA & Q9QU10 & RHOA & 1.964 \\
\hline 24 & Transport and Golgi organization 2 homolog & P54797 & TANG02 & 1.514 \\
\hline 25 & Fructose-bisphosphate aldolase & A6Zl44 & ALDOA & 1.549 \\
\hline 26 & Tripeptidyl-peptidase 1 & 089023 & TPP1 & 1.457 \\
\hline 27 & $\begin{array}{l}\text { Pyruvate dehydrogenase E1 component subunit } \\
\text { alpha, testis-specific form }\end{array}$ & P35487 & PDHA2 & 1000 \\
\hline 28 & Mitochondrial thiamine pyrophosphate carrier & Q9DAM5 & SLC25A19 & 0.645 \\
\hline 29 & Solute carrier family 35 member F6 & Q8VE96 & SLC35F6 & 1.577 \\
\hline 30 & Ribonuclease P 14 subunit (Human) & J3QMX0 & RPP14 & 0.453 \\
\hline 31 & Coiled-coil domain-containing protein 58 & Q8R3Q6 & CCDC58 & 1.907 \\
\hline 32 & $\begin{array}{l}\text { Haloacid dehalogenase-like hydrolase domain- } \\
\text { containing protein 3 }\end{array}$ & Q9CYW4 & HDHD3 & 0.602 \\
\hline 33 & tRNA methyltransferase 10 homolog C & Q3UFY8 & TRMT10C & 1.503 \\
\hline 34 & Deaminated glutathione amidase & Q8VDK1 & NIT1 & 0.571 \\
\hline 35 & LETM1 domain-containing protein 1 & Q924L1 & LETMD1 & 0.656 \\
\hline 36 & Aurora kinase A-interacting protein & Q9DCJ7 & AURKAIP1 & 1.610 \\
\hline & & & & \\
\hline
\end{tabular}

Table 2. Primer sequences for qRT-PCR.

\begin{tabular}{|ll|}
\hline Gene & Primer Sequence \\
PDHE1a & Forward 5'-GGG ACG TCT GTT GAG AGA GC-3' \\
& Reverse 5'-TGT GTC CAT GGT AGC GGT AA-3' \\
PDK4 & Forward 5'-CAG CTG GTG AAG AGC TGG TA-3' \\
& Reverse 5'-CTC TGA CAG GGC TTT CTG GT-3' \\
\hline
\end{tabular}

\section{Figures}


A

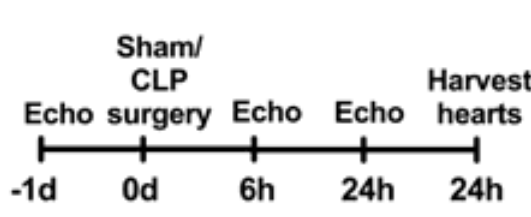

Experimental timeline
B

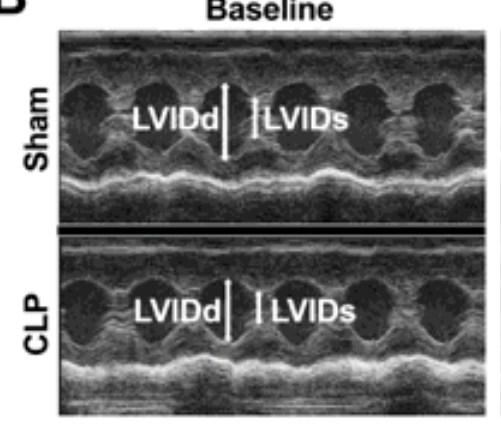

24 hours

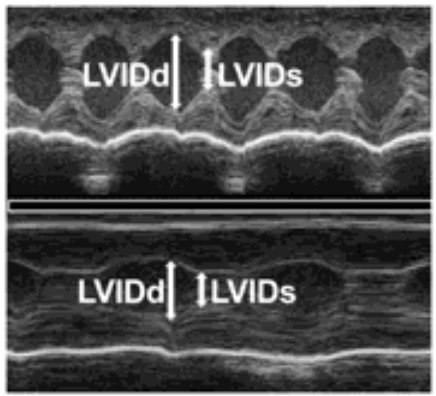

Heart Rate

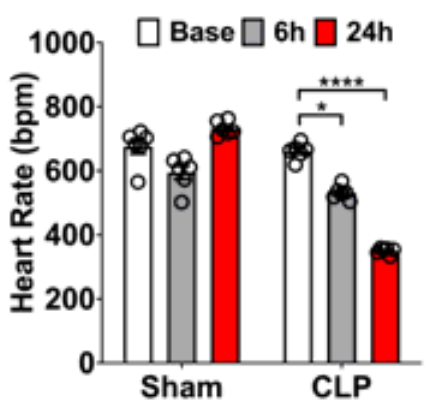

LVIDs

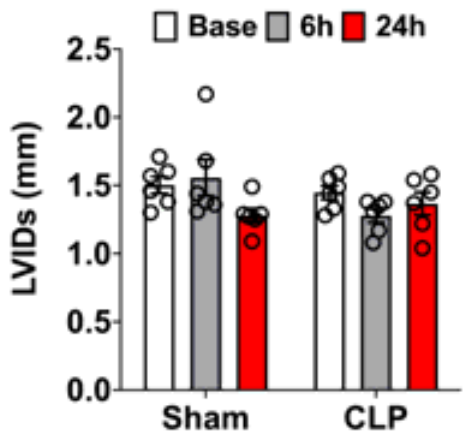

C

Cardiac Output

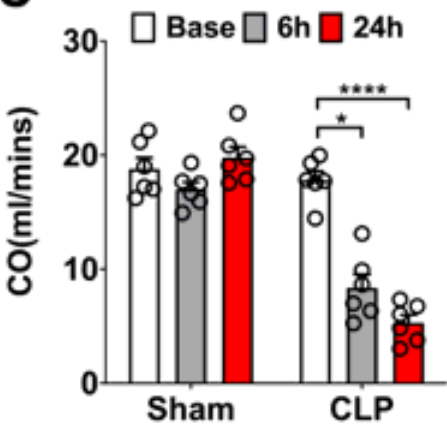

\%Fractional Shortening

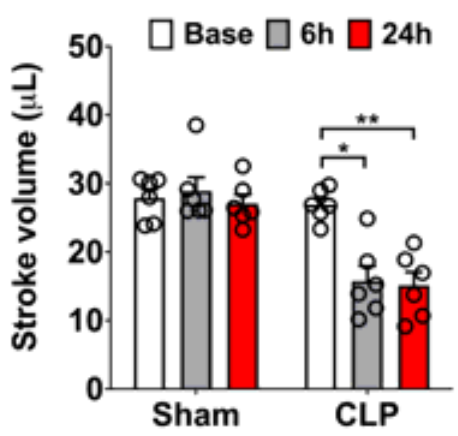

LVIDd
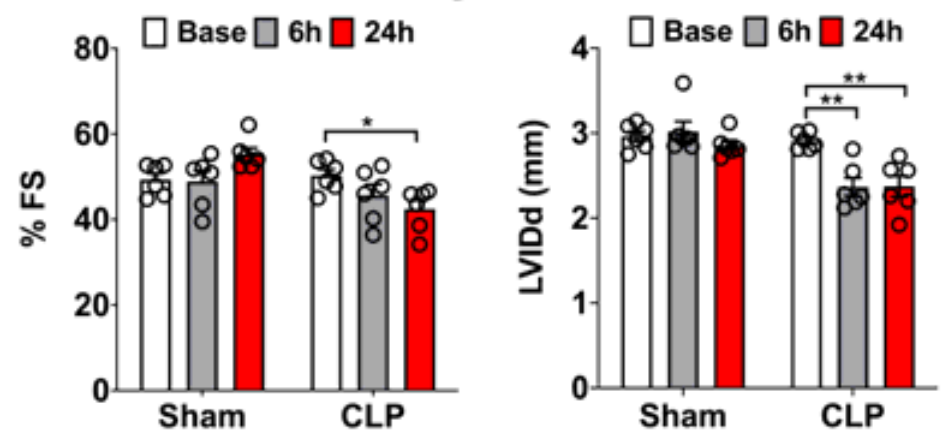

LVDP

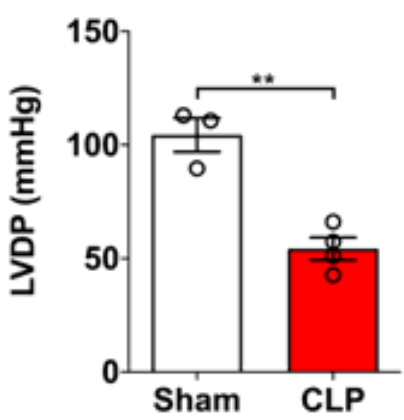

$\mathrm{dP} / \mathrm{dT}$

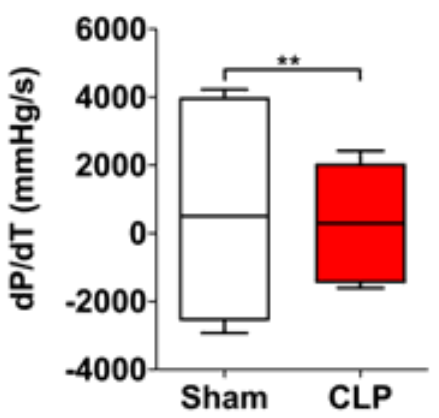

Figure 1

Cardiac dysfunction in sepsis mice. A. The timeline of experiments. A baseline echocardiography (Echo) was performed one day prior to surgical procedures. The next day, sham (laparotomy only) or CLP (laparotomy plus cecal ligation and puncture) surgery was performed and Echo used to assess cardiac function at $6 \mathrm{~h}$ and $24 \mathrm{~h}$ post-surgery. After the $24 \mathrm{~h}$ Echo, animals were sacrificed, and mitochondria were isolated for subsequent experiments. B. Representative Echo M-mode images showing LVIDd/LVIDs at 
baseline and $24 \mathrm{~h}$ after sham or CLP procedure. C. Echo measurements at baseline and at $6 \mathrm{~h}$ and $24 \mathrm{~h}$ after sham or CLP surgery. $n=6 /$ group. $D$. Ex vivo Langendorff-perfused hearts. Left ventricular (LV) developed pressure (LVDP) and rate of LVDP (dP/dT) were measured at 24h after sham and CLP procedures. $n=3-4 /$ group. Each error bar represents mean \pm SEM. Data in $C$ were analyzed using 2-way ANOVA with Sidak's multiple comparisons test. Panels in $\mathrm{D}$ were analyzed using two-tailed t test. ${ }^{*} \mathrm{P}<$ $0.05,{ }^{*} \mathrm{P}<0.01,{ }^{*} \mathrm{P}<0.001$.

A

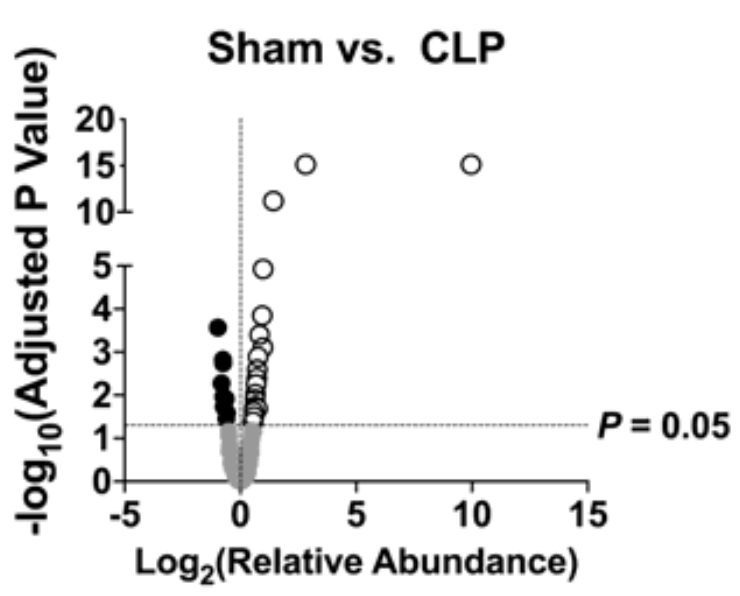

B
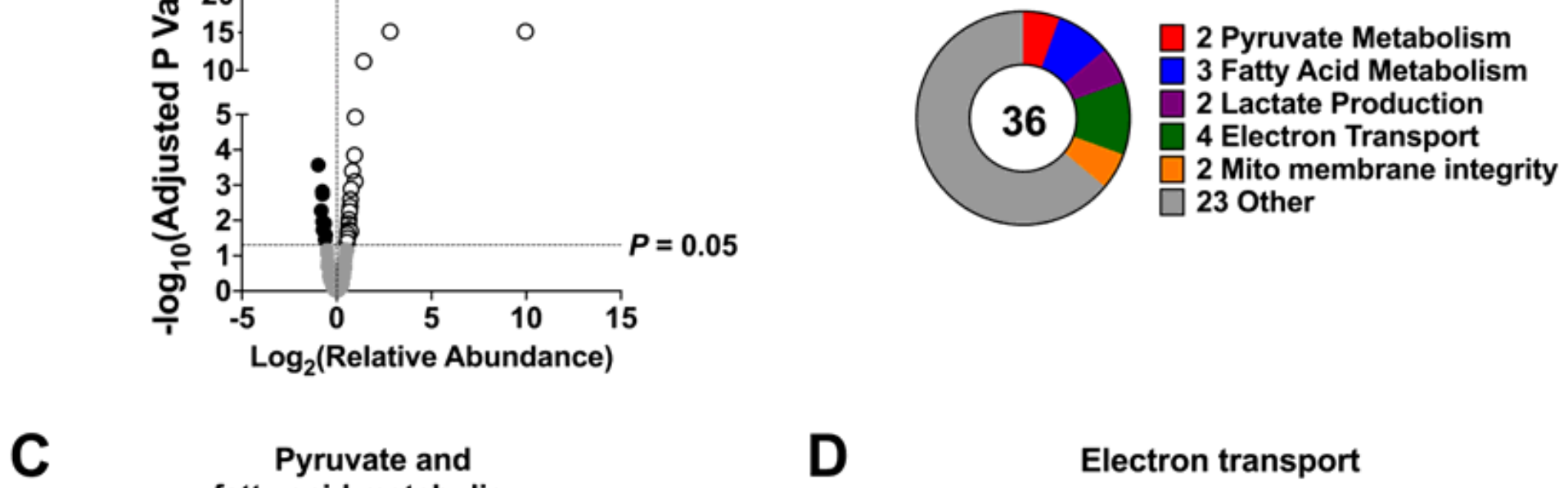

Pyruvate and fatty acid metabolism

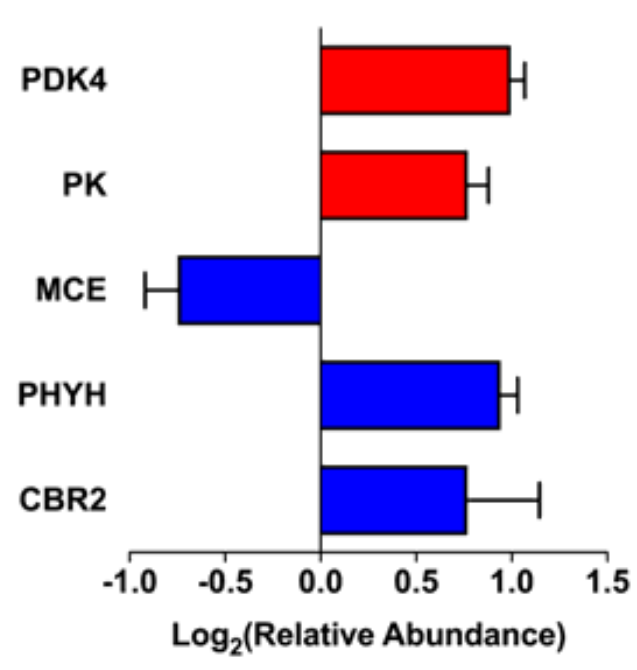

D

Electron transport

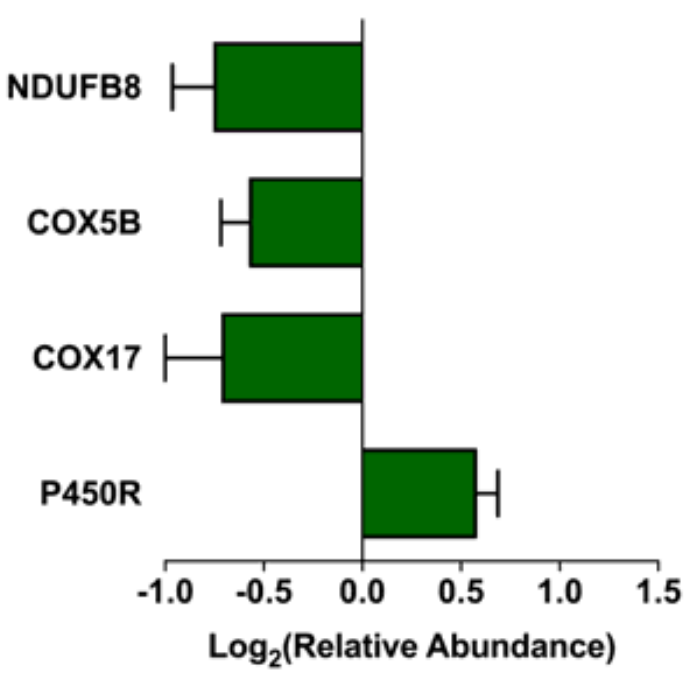

Figure 2

Proteomic analysis of isolated cardiac mitochondria from septic mice. Mitochondria were isolated from the hearts of sham- and CLP-operated mice and proteomic analysis was conducted via liquid chromatography-tandem mass spectrometry. A. Volcano plot of all mitochondrial or metabolically coupled proteins found in the isolated mitochondria. All proteins detected by the mass spectrometry were referenced against a mouse reference proteome using a Sequest HT algorithm and MS Amanda algorithm as described in Methods. Proteins considered to be non-mitochondrial or non-metabolically related based on their identity were excluded from the plot. Proteins with P-values less than 0.05 were 
considered significant changes between the two groups. Black dots represent decreased expression while white open dots indicate increased expression. B. Proteins, total 35 differentially expressed in sepsis mitochondria, are grouped in different colors based on their roles in glycolysis, fatty acid metabolism, electron transport, or mitochondria membrane integrity. C-D. List of mitochondrial specific proteins with altered expression that are involved in either pyruvate metabolism, fatty acid metabolism, or electron transport. Each color is corresponding to different metabolic pathway as shown in B. Each mouse heart was assessed individually by the proteomics and was not mixed together. $n=6$ mitochondrial preparations from each group were used for proteomic analysis. Statistical analysis was done as described in the methods section. (Abbreviations: PDK4, pyruvate dehydrogenase kinase 4, PK, pyruvate kinase, CBR2, carbonyl reductase [NADPH] 2, PHYH, Phytanoyl-CoA 2-Hydroxylase, MCE, methylmalonylCoA epimerase, NDUFB8, NADH ubiquinone oxioreductase subunit B8, COX5B, cytochrome c oxidase 5B, COX 17, cytochrome c oxidase copper chaperone, P450R, NADPH-cytochrome P450 reductase).

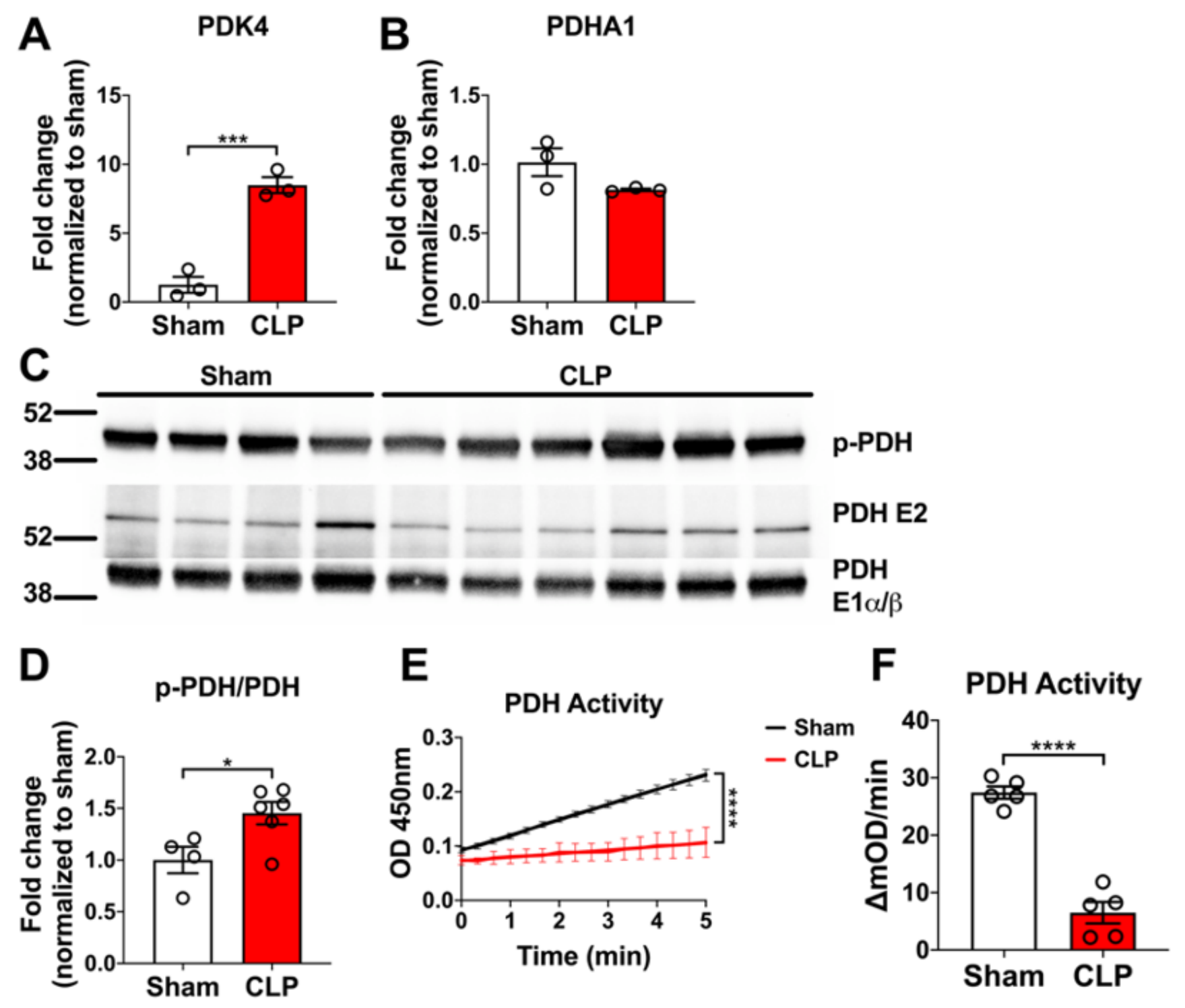

Figure 3 
Functional and molecular analysis of PDH in septic hearts. A-B. qRT-PCR analysis of myocardial PKD4 and PDH1Ea gene expression, respectively, in sham or CLP mice. Each dot corresponds to one mouse heart $(n=3)$. C. Western blot of serine 293-phosphorylated ( $p-P D H)$ and total PDH in whole hearts from sham and sepsis mice. PDH antibody detects two bands, E2 and E1 $\alpha / \beta$. The uncropped Western blot images were presented in Supplemental Figure 1 and Supplemental Figure 2. The E2 bands were cropped from E1 bands and adjusted for optimal exposure. D. Densitometry analysis was done using Image J software and p-PDH levels were normalized to total PDH levels. $n=4$ (sham), 6 (CLP) mice. E.

Quantification of PDH activity over time. F. Bar graph quantifying the change in PDH activity per min. PDH activity was calculated using the formula described in Methods. $n=5 /$ group. Each error bar represents mean \pm SEM. For all panels, data was analyzed using two-tailed t-test. ${ }^{*} P<0.05,{ }^{* \star *} P<0.001$, $\star \star \star \star P<0.0001$ (Abbreviations: PDK4, pyruvate dehydrogenase kinase 4, PDHE1a, pyruvate dehydrogenase E1 subunit a, PDH, pyruvate dehydrogenase).
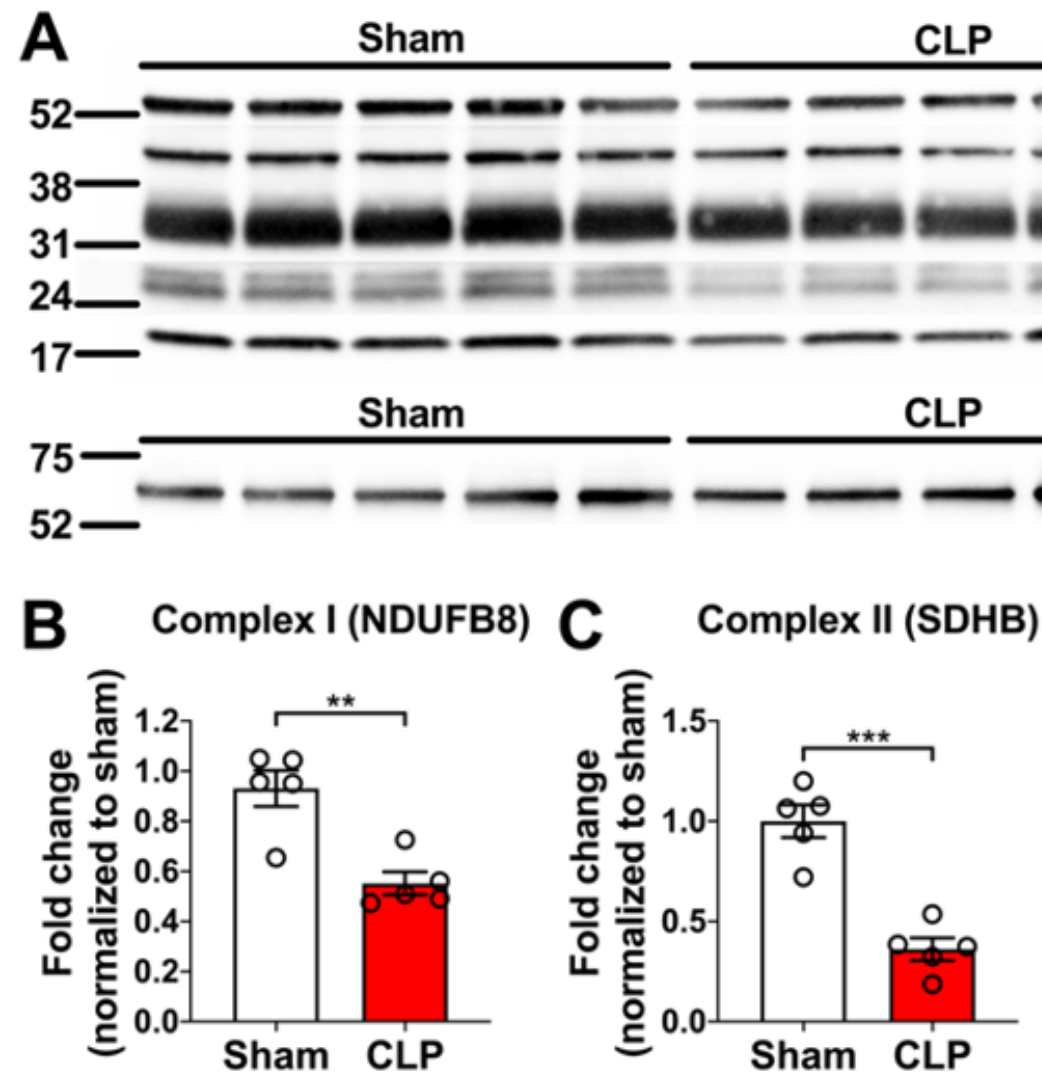

D Complex III (UQCRC2)
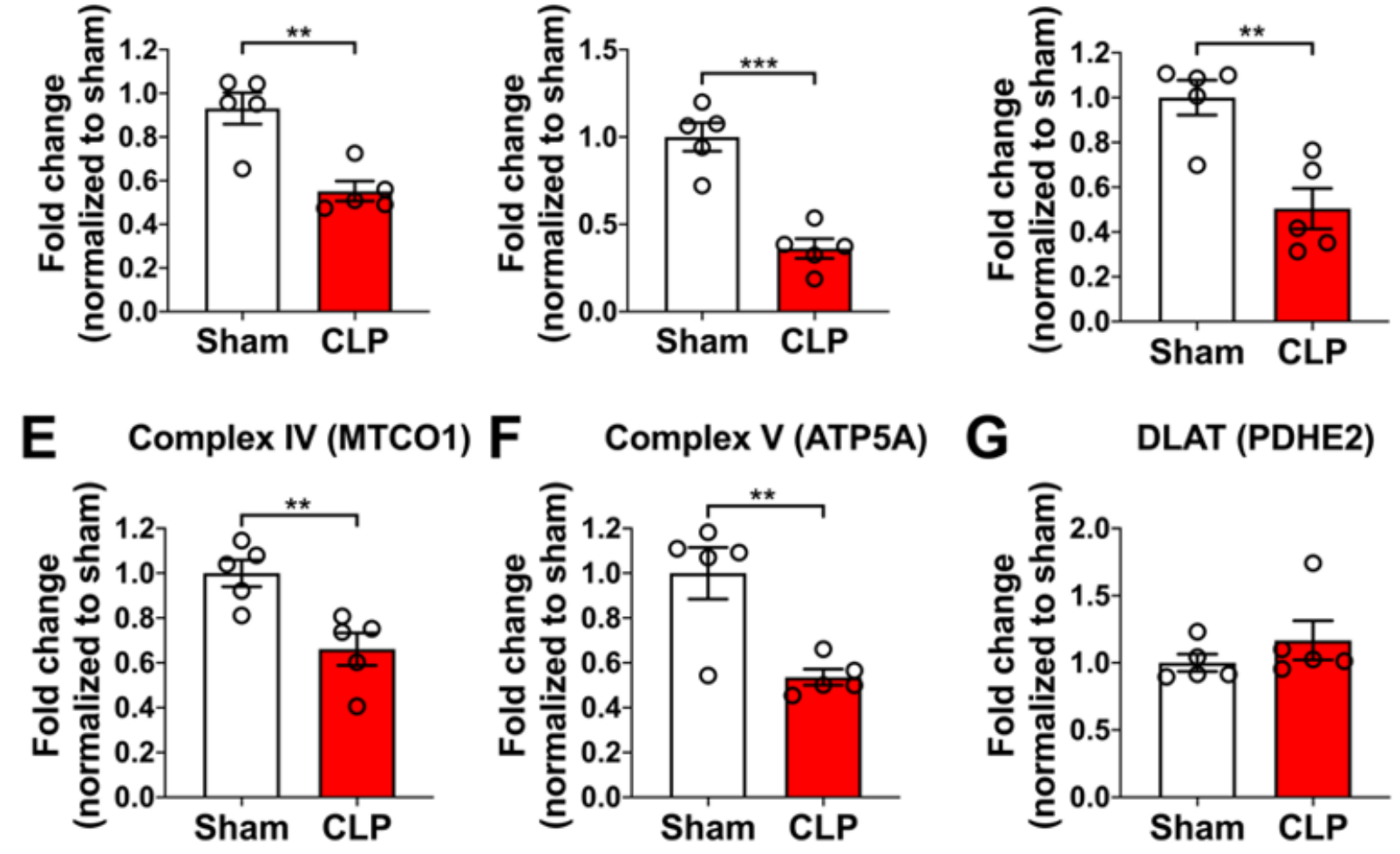


\section{Figure 4}

Molecular analysis of the ETC (complexes I-V) in septic mitochondria. A. Western blot of isolated cardiac mitochondria showing decreased expression of representative subunits from all five oxidative phosphorylation complexes but not of an unrelated protein, DLAT. The uncropped images of the Western blots were presented in Supplemental Figure 3 and Supplemental Figure 4. The images of each complex and DLAT Western blot were separately developed and adjusted for optimal exposure. B-G. Densitometry analysis was done using ImageJ software and fold change for each protein was normalized to PDHE2 levels before comparison with sham. $n=5$ /group. Each error bar represents mean \pm SEM. For all panels, data was analyzed using two-tailed t-test. ${ }^{*} P<0.05,{ }^{*} \mathrm{P}<0.01 * \star \star \mathrm{P}<0.001$. (Abbreviations: ATP5A, ATP synthase F1 subunit alpha, UQCRC2, ubiquinol-cytochrome c reductase core protein 2, MTCO1, mitochondrially encoded cytochrome $c$ oxidase $1, S D H B$, succinate dehydrogenase complex iron sulfur subunit B, NDUFB8, NADH-ubiquinone oxioreductase subunit B8, DLAT, dihydrolipoamide Sacetyltransferase). 
A

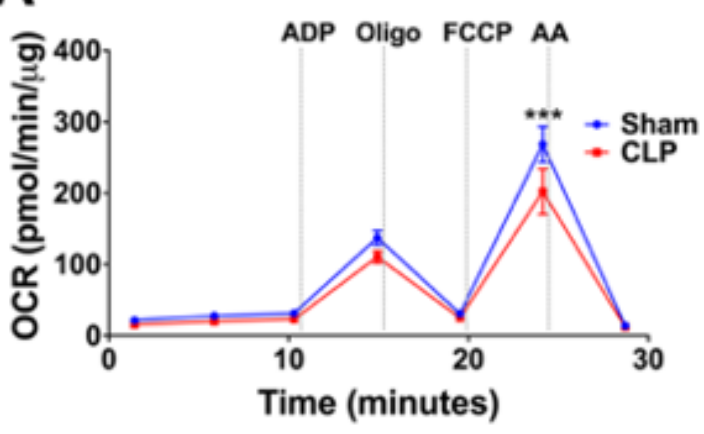

B

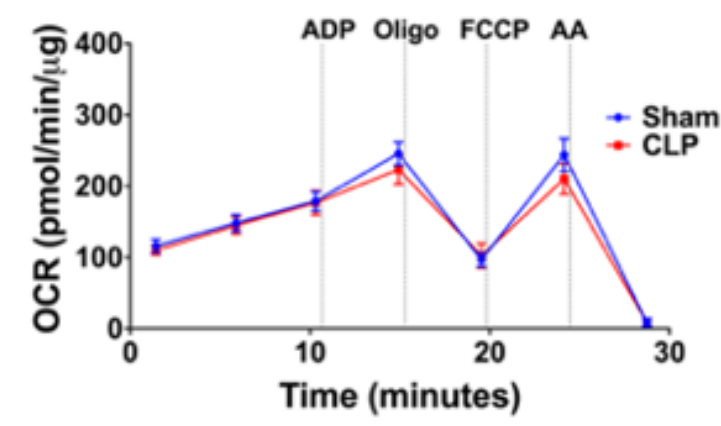

Basal respiration
Pyruvate/Malate

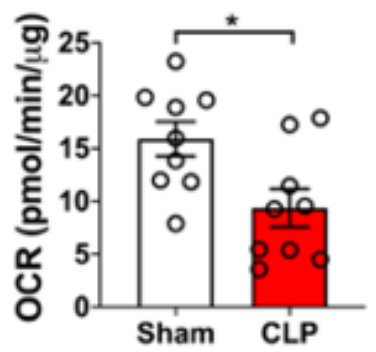

G
D $\quad \begin{gathered}\text { State III } \\ \text { Pyruvate/Malate }\end{gathered}$

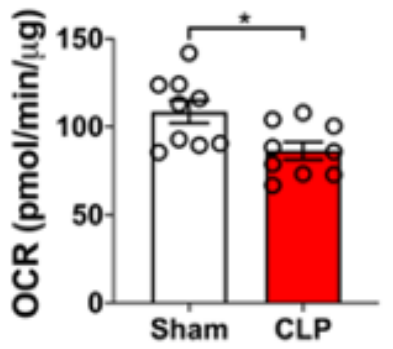

H

State III Succinate/Rotenone

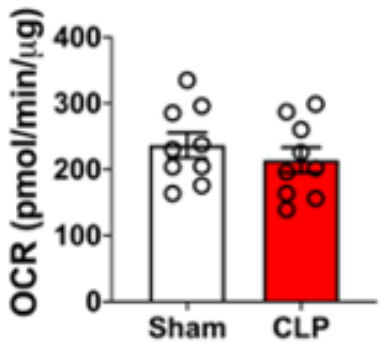

E $\quad \begin{gathered}\text { State IV } \\ \text { Pyruvate/Malate }\end{gathered}$

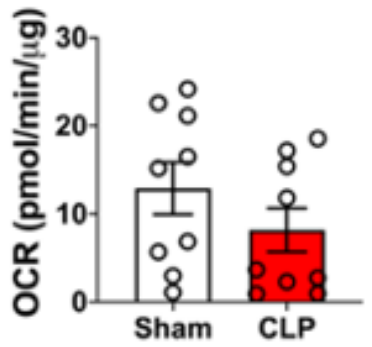

State IV Succinate/Rotenone

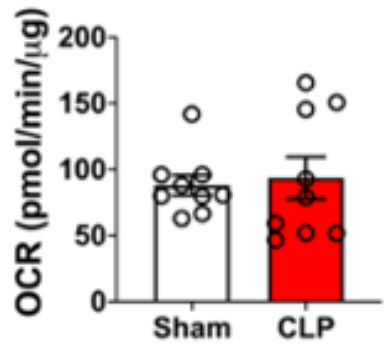

M
State III Palmitoyl-Carnitine/Malate
State IV

Palmitoyl-Carnitine/Malate
F State III Pyruvate/Malate Palmitoyl-Carnitine/Malate
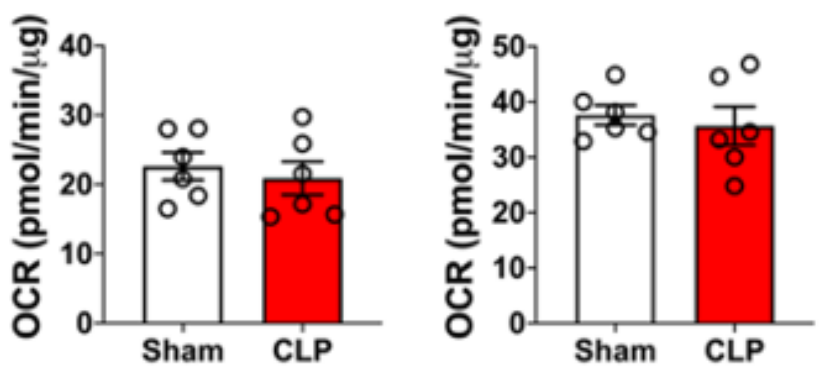

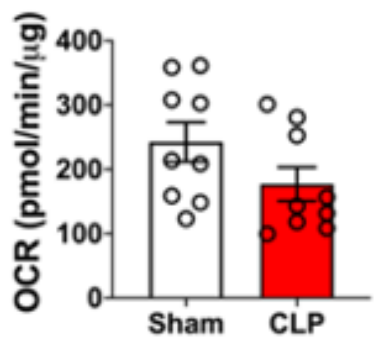

State $\mathrm{III}_{\mathrm{u}}$ Succinate/Rotenone

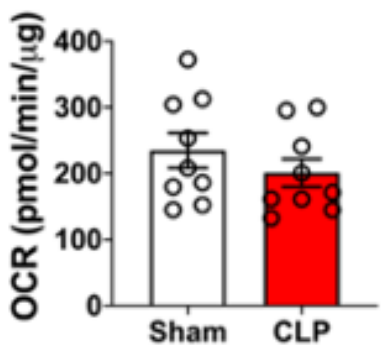

State III Palmitoyl-Carnitine/Malate

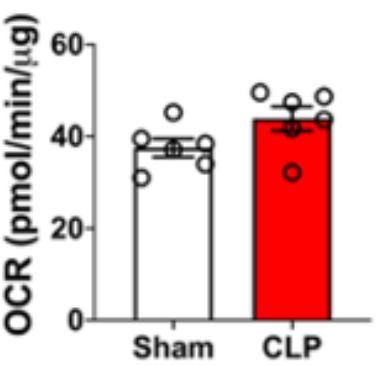

Figure 5

Oxygen consumption by cardiac mitochondria of sham and sepsis mice. Heart mitochondria were isolated from sham or CLP mice $24 \mathrm{~h}$ after surgery. A-B. Isolated cardiac mitochondria were given the Complex Ilinked substrates pyruvate and malate or the Complex II substrate succinate in the presence of the Complex I inhibitor rotenone. Oxygen consumption was measured before and after the sequential additions of ADP, oligomycin, FCCP, and antimycin A. C-F. Oxygen consumption rate (OCR) for sham and 
CLP mitochondria during basal (basal - antimycin A), state 3 (ADP - antimycin A), state 4 (oligomycin antimycin A), and state 3 uncoupled (state $3 \mathrm{u}$, FCCP - antimycin A) respiration in the presence of pyruvate and malate, succinate and rotenone, or palmityolcarnitine and malate. Each dot represents mitochondrial respiration data from a single mouse preparation. $n=6-9$ mice per group. Each error bar represents mean \pm SEM. A-B were analyzed using two-way ANOVA with Sidak's multiple comparison test. For $\mathrm{C}-\mathrm{N}$, data were analyzed using two-tailed t-test. ${ }^{*} \mathrm{P}<0.05$. (Abbreviations: $A D P$, adenosine diphosphate, oligo, oligomycin, AA, antimycin A).

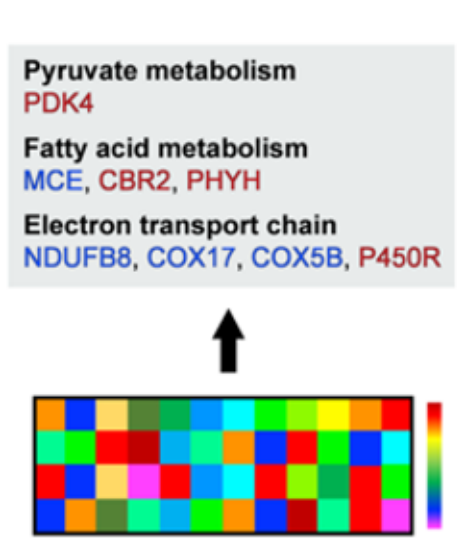

Proteomics

\section{Sepsis}
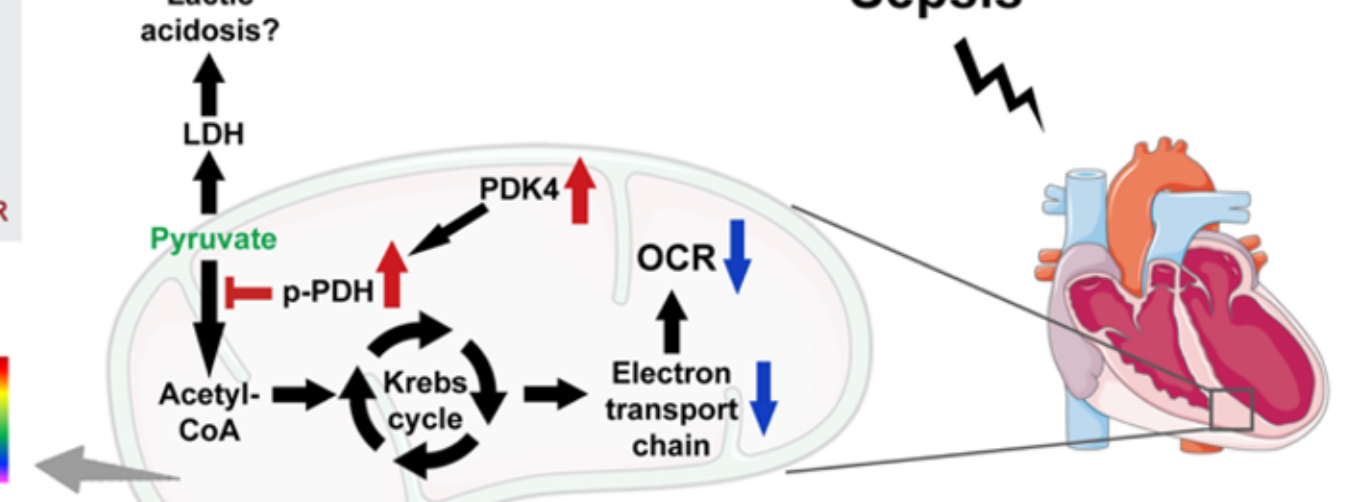

Cardiomyopathy

Figure 6

Molecular remodeling of cardiac mitochondrial in murine sepsis. Proteomic analysis of mitochondria from hearts with sepsis-induced cardiomyopathy revealed several mitochondria-specific proteins with altered expression including PDK4 and the electron transport chain proteins NDUFB8, COX17, COX5B, and P450R. Increase in PDK4 expression leads to phosphorylation and inactivation of the pyruvate metabolizing enzyme, PDH. Diminished PDH activity, along with the decreased expression of several electron transport chain proteins, reduces the overall capacity of the mitochondria to utilize the critical metabolic substrate pyruvate resulting in a lower oxygen consumption rate as shown by the schematic above. These molecular modifications may provide insight into how the mitochondria is linked to the development of sepsis-induced cardiomyopathy. (Abbreviations: PDK4, pyruvate dehydrogenase kinase 4; $\mathrm{MCE}$, methylmalonyl-CoA epimerase; CBR2, carbonyl reductase [NADPH] 2; PHYH, Phytanoyl-CoA 2Hydroxylase; NDUFB8, NADH ubiquinone oxioreductase subunit B8; COX 17, cytochrome c oxidase copper chaperone; COX5B, cytochrome c oxidase 5B; P450R, NADPH-cytochrome P450 reductase; OCR, oxygen consumption rate; $L D H$, lactate dehydrogenase; $\mathrm{p}-\mathrm{PDH}$, phospho-pyruvate dehydrogenase).

\section{Supplementary Files}

This is a list of supplementary files associated with this preprint. Click to download. 
Page 26/26 